\title{
EVALUACIÓN DE LA VULNERABILIDAD DEL SISTEMA DE PREVENCIÓN CONTRA INCENDIO DEL CAMPUS DE LA UNIVERSIDAD DEL QUINDIO
}

\author{
VULNERABILITY EVALUATION OF FIRE PREVENTION \\ SYSTEM OF THE UNIVERSITY OF QUINDIO
}

Yelixza Hernandez ${ }^{1}$, Paula Toro ${ }^{1}$ y Elkin Monsalve ${ }^{2}$

${ }^{1}$ Estudiantes Programa de Ingeniería Civil, Universidad del Quindío

2. CEIFI, Grupo CIDERA - Facultad de Ingeniería. Universidad del Quindío

Recibido: 10 de febrero de 2014

Aceptado: 16 de marzo de 2014

* Correspondencia: Facultad de Ingeniería. Universidad del Quindío, e-mail: elkinmonsalve@uniquindio.edu.co

\begin{abstract}
RESUMEN
El estudio plantea evaluar la vulnerabilidad del sistema de protección contra incendios de las edificaciones del campus de la Universidad del Quindío, siguiendo los requerimientos establecidos en el año 2010 en los títulos $\mathrm{J}$ "Requerimientos de protección contra incendios en edificaciones" y K "Requisitos complementarios" de la Norma Sismo Resistente de Colombia. De acuerdo al resultado de la evaluación, se definen las alternativas de solución a emplear. En este el artículo se presenta, de igual manera, el procedimiento a realizar para evaluar el estado y la capacidad de acción que puede tener una Institución de Educación Superior según las características de sus edificaciones.
\end{abstract}

Palabras claves: Clave: Sistema contra incendio, vulnerabilidad de edificaciones, titulo J y K, NSR 2010, campus universitario.

\begin{abstract}
The study proposes to evaluate the vulnerability of the fire protection system of the buildings of the University of Quindío following the requirements established in 2010 seen in the titles J "fire protection requirements in buildings" and K "Additional requirements" of Colombia Earthquake Resistant Standards. According to the result of the evaluation, alternative solutions are defined to use. In addition, the evaluation procedures implemented to measure the status and capacity of action of an institution of higher education is presented according to the characteristics of its buildings.
\end{abstract}

Keywords: Fire protection system, building vulnerability, title J \& K, NSR 2010, campus. 


\section{INTRODUCCION}

El riesgo se encuentra asociado a las pérdidas y daños relacionados con las distintas actividades humanas. El riesgo es una condición latente que, al no ser modificada o mitigada a través de la intervención humana o por medio de un cambio en las condiciones del entorno físico-ambiental, anuncia un determinado nivel de impacto social y económico hacia el futuro, cuando un evento físico detona o actualiza el riesgo existente.

Son entonces factores de riesgo, la amenaza y la vulnerabilidad; siendo esta última, la que tiene relación con la preparación y disposición (humana, medios, elementos, etc) para soportar alteraciones (daños, pérdidas, afectaciones, etc) frente a la ocurrencia de un evento potencialmente peligroso.

Para el caso de las edificaciones, estas pueden estar sometidas a diversas amenazas y, a diferente nivel (sismo, vendavales, huracanes, inundaciones, incendios, entre otros). La predisposición a sufrir daños y pérdidas varía de acuerdo al evento físico. El estar preparados, analizando y siendo conocedores de la amenaza y vulnerabilidad, permite enfrentare las perturbaciones parciales o totales que se puedan dar en una edificación y disminuir el riesgo de quienes habitan o transitan por ellas. Para el caso de Instituciones de Educación Superior, como la Universidad del Quindío, se cuenta con un campus constituido por edificaciones de carácter administrativo, académico, deportivo, investigativo, etc, donde se movilizan continuamente estudiantes, profesores, empleados y comunidad en general.

Las edificaciones del campus universitario, no están exentas de algún tipo de conflagración, que puede provocar múltiples desastres e innumerables pérdida de vidas, si no se implementa adecuadamente una red o medidas que brinden protección tanto en el interior, como en el exterior de las edificaciones que lo componen.

Con el objetivo de evaluar la vulnerabilidad del sistema de protección contra incendios y plantear soluciones, se realizó un estudio en el campus de la Universidad del Quindío caracterizando las diferentes edificaciones y evaluándolas según los parámetros establecidos en los títulos J "Requerimientos de protección contra incendios en edificaciones" y K "Requisitos complementarios" de la Norma Sismo Resistente de Colombia del año 2010 (2-3). Es además objeto de este artículo, presenta el reporte de caso del estudio y procedimientos realizados para evaluar el estado y la capacidad de acción que puede tener una Institución de Educación Superior con edificaciones construidas con anterioridad a la normatividad reciente.

\section{MATERIALES Y MÉTODOS \\ Referentes Conceptuales}

El estudio se desarrolló en el marco de diferentes políticas y referentes internacionales y nacionales asociadas a la Gestión de Riesgo de Desastres. Se toma como base fundamental, que dentro de los propósitos que se plantean para contribuir a alcanzar los objetivos del milenio, las infraestructuras de los centros educativos tanto como sus instalaciones sanitarias, agua y saneamiento sean resistentes a catástrofes (4).

El desarrollo del tema en las instituciones educativas, se sustenta en tres ejes: la seguridad interna, la formación de los estudiantes en gestión del riesgo a desastres y el compromiso de las instituciones educativas y del sector educación con la reducción de riesgos, el manejo de los eventos adversos y la recuperación de las comunidades y del país (5). Se plantea entonces que realmente los centros educativos deben hacer gestión de riesgo, promoviendo el interés para que las Comunidades Educativas cuenten con instalaciones más seguras. El Plan Nacional para la Prevención y Atención de Desastres, que expidió el gobierno nacional de Colombia mediante el decreto 93 de 1998, tiene como objeto orientar las acciones del Estado y de la sociedad civil para la prevención y mitigación de riesgos, los preparativos para la atención y recuperación en caso de desastre, contribuyendo a reducir el riesgo y al desarrollo sostenible de las comunidades vulnerables ante los eventos naturales y antrópicos (6).

Para emprender el estudio de evaluación de la vulnerabilidad del sistema de prevención contra incendio del campus, es necesario establecer que la Amenaza es el peligro latente de que un evento físico de origen natural, o causado, o inducido por la acción humana de manera accidental, se presente con una severidad suficiente para causar pérdida de vidas, lesiones u otros impactos en la salud, así como también daños y pérdidas en los bienes, la infraestructura, los medios de sustento, la prestación de servicios y los recursos ambientales.(7). Este evento físico a tener presente en el estudio está representado por la posibilidad de que 
se presenten incendios. Para el caso de la Vulnerabilidad, esta es definida (7) como la Susceptibilidad o fragilidad física, económica, social, ambiental o institucional que tiene una comunidad de ser afectada o de sufrir efectos adversos en caso de que un evento físico peligroso se presente. Corresponde a la predisposición a sufrir pérdidas o daños de los seres humanos y sus medios de subsistencia, así como de sus sistemas físicos, sociales, económicos y de apoyo que pueden ser afectados por eventos físicos peligrosos.

La vulnerabilidad corresponde a la predisposición o susceptibilidad que tiene un elemento a ser afectado o a sufrir una pérdida. En consecuencia, la diferencia de vulnerabilidad de los elementos determina el carácter selectivo de la severidad de los efectos de un evento externo sobre los mismos. Un análisis de vulnerabilidad es un proceso mediante el cual se determina el nivel de exposición y la predisposición a la pérdida de un elemento o grupo de elementos ante una amenaza específica, contribuyendo al conocimiento del riesgo a través de interacciones de dichos elementos con el ambiente peligroso. (8).

Para establecer las condiciones en que se encuentran los edificios del campus universitario frente a un incendio, se toman como referencia los títulos J y K de la NSR 2010. El Título J "Requisitos de Protección Contra Incendios en Edificaciones" hace referencia a los elementos y especificaciones de tipo constructivo que deben tener las edificaciones para prevenir y controlar un incendio, ya sea al inicio o en su pro-

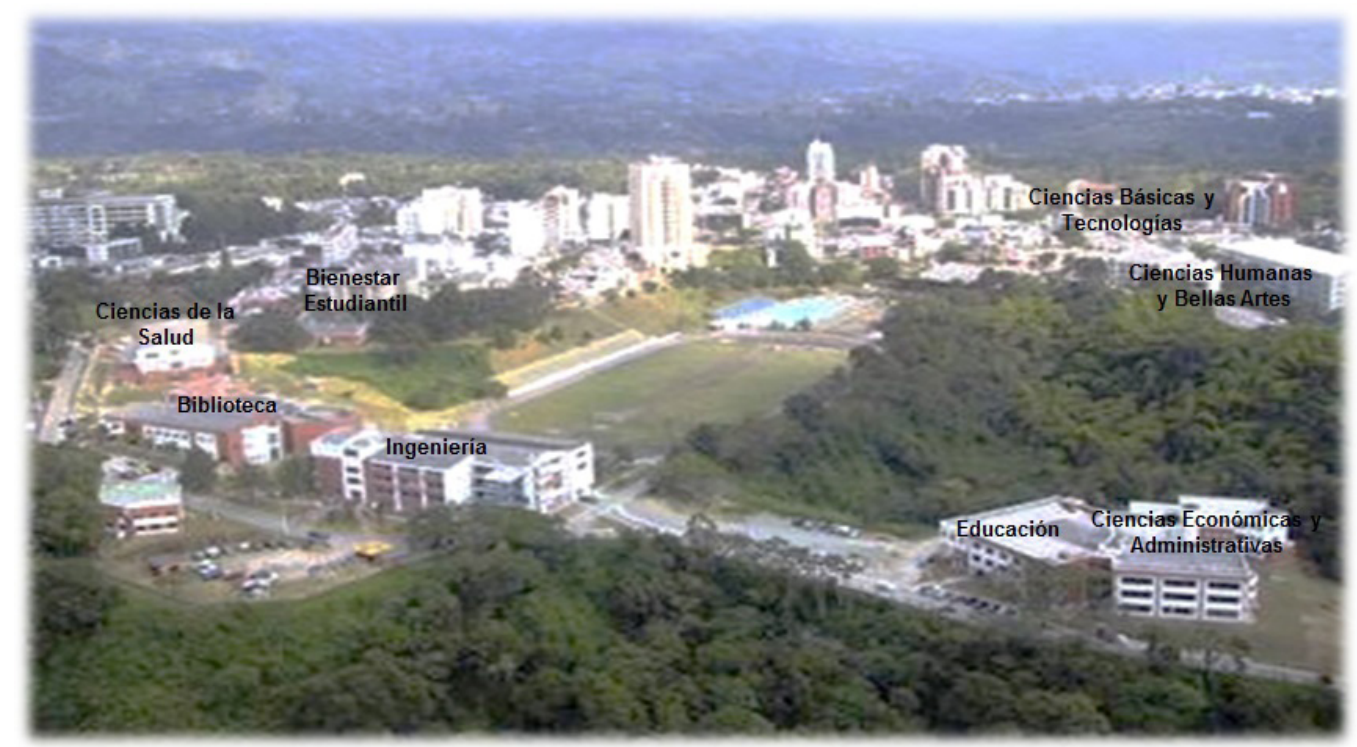

Figura. 1 Campus Universidad del Quindío longación.; para esto, la información a caracterizar o levantar tiene relación con los requerimientos que deben poseer las diferentes clases de elementos presentes en un sistema contra incendios. Estos comprenden tomas fijas para bomberos, gabinetes contra incendios y extinguidores; también se incluyen especificaciones estructurales en el caso de muros cortafuegos, materiales en cuanto a fachas y muros internos y de elementos como escaleras y puertas de acceso. Para el caso de el Titulo K "Requisitos Complementarios", se deben tener presente los requisitos complementarios que dispondrán las edificaciones para disminuir la vulnerabilidad ante un incendio; entre ellos la señalización de accesos y lugares para personas discapacitadas, la iluminación y todo lo concerniente a los núcleos de evacuación a implementar en todos los establecimientos.

\section{Zona de Estudio}

El trabajo fue desarrollado en el campus de la Universidad del Quindío, específicamente en los edificios donde funcionan las facultades de Ciencias Básicas y Tecnológicas, Bellas Artes, Ciencias de la Salud, Ingeniería, Ciencias Económicas y Administrativas, Educación y Ciencias Agroindustriales. De igual manera en las edificaciones, como; la biblioteca Euclides Jaramillo Arango, biblioteca Infantil, salas de estudio, capilla, coliseo, centro audiovisuales, plantas piloto, laboratorio de biomédicas, bienestar universitario y bloques administrativos I y II. En la Figura. 1 donde se presenta la panorámica de la Universidad del Quindío, se observan las diferentes edificaciones evaluadas. 


\section{Trabajo de campo}

Para la caracterización de las edificaciones se establecieron instrumentos de recolección de información que miden los parámetros, aspectos y requerimientos relacionados en los títulos J y K de la NSR 2010. En la Figura. 2 se observa el instrumento de recolección de información empleado. La información recolectada permite, de acuerdo a los parámetros de norma establecer el estado actual de las diferentes edificaciones.



Figura. 2 Formato de Recolección de Información Títulos J y K. (NSR, 2010)

Paralelo a la evaluación de las edificaciones, se requiere establecer el estado de los elementos de protección contra incendios y el funcionamiento hidráulico de los hidrantes ubicados en el campus. El Cuerpo Oficial de Bomberos de la ciudad de Armenia prestó el soporte técnico en la visita a cada edificación; y con las Empresas Públicas de Armenia se realizó un recorrido por los diferentes hidrantes con el fin de realizar pruebas de toma de presiones y funcionamiento de los mismos.

\section{Procesamiento y Análisis de la información}

Procesar y analizar la información requirió un conocimiento total de los siguientes parámetros: Titulo J; requisitos de acceso a la edificación, la prevención de la propagación del fuego hacia el exterior y el interior, los acabados interiores, la clasificación de edificaciones en función del riesgo de pérdida de vidas humanas o amenaza de combustión, la resistencia requerida contra el fuego, los sistemas y equipos para detección y alarma de incendios, como la detección y extinción de los mismos. Del título K: analizar lo relacionado con la localización y mantenimiento, la señalización e iluminación, las alarmas, los sistemas de evaluación para discapacitados, la carga de ocupación, el factor de carga de ocupación, la capacidad de las salidas, el acceso y recorrido a las salidas, los medios de evacuación, la iluminación de los medios de evacuación, como su señalización.

Para la aplicación de los requisitos de los títulos $\mathrm{J}$ y $\mathrm{K}$ es necesaria la clasificación de las edificaciones por grupos de ocupación, la cual se realiza según la Tabla J.1.1-1 y Tabla K.2.6.3 de la NSR 2010 como se observa en la Figura. 3 y Figura. 4 respectivamente. Al encontrar el subgrupo de ocupación (I-3) por ser su clasificación institucional (K.2.6), y de educación por tenerse espacios o edificaciones empleados para la reunión de personas con propósitos educativos y de institución, se procede a la clasificación de edificaciones en función del riesgo de pérdida de vidas humanas o amenaza de combustión.

\section{RESULTADOS Y DISCUSIÓN}

Al relacionar la información que describe la situación física y de funcionamiento de cada edificación con los parámetros de los Títulos J y K se encontraron diversas situaciones, las cuales fueron sujeto de observaciones, comentarios y recomendaciones. En la Figura. 5 se observa un ejemplo para la evaluación de accesos y en la Figura. 6 se observa un ejemplo de rutas de evacuación

En la visita de evaluación de las edificaciones, el Cuerpo Oficial de Bomberos de Armenia estableció los sitios en los cuales deben estar ubicados los extintores, los tipos de extintores a emplear, la manipulación de los mismos. De igual manera realizó recomendaciones en algunos lugares sobre el retiro de material combustible y los elementos que obstaculizan la evacuación ante el caso de una emergencia. 


\begin{tabular}{|c|c|c|}
\hline $\begin{array}{l}\text { Grupos y Subgru- } \\
\text { pos de Ocupación }\end{array}$ & Clasificación & $\begin{array}{l}\text { Sección del } \\
\text { Reglamento }\end{array}$ \\
\hline $\bar{A}$ & AMACENAMIENTO & K.2.2 \\
\hline$A-1$ & Riesgo moderado & \\
\hline$A-2$ & Riesgo bajo & \\
\hline $\mathrm{C}$ & COMERCIAL & K.2.3 \\
\hline $\mathrm{C}-1$ & Servicios & \\
\hline $\mathrm{C}-2$ & Bienes & \\
\hline$E$ & ESPECIALES & K.2.4 \\
\hline$F$ & FABRIL E INDUSTRIAL & K.2.5 \\
\hline F-1 & Riesgo moderado & \\
\hline $\mathrm{F}-2$ & Riesgo bajo & \\
\hline I & INSTITUCIONAL & K.2.6 \\
\hline |-1 & Reclusión & \\
\hline $1-2$ & Salud o incapacidad & \\
\hline $1-3$ & Educación & \\
\hline $1-4$ & Seguridad pública & \\
\hline $1-5$ & Servicio público & \\
\hline$L$ & $\begin{array}{l}\text { LUGARES DE } \\
\text { REUNIÓN }\end{array}$ & K.2.7 \\
\hline$L-1$ & \begin{tabular}{|l} 
Deportivos \\
\end{tabular} & \\
\hline $\bar{L}-2$ & Culturales y teatros & \\
\hline$L-3$ & Sociales y recreativos & \\
\hline$L-4$ & Religiosos & \\
\hline $\bar{L}-5$ & De transporte & \\
\hline $\bar{M}$ & MIXTOS UOTROS & $K .2 .8$ \\
\hline $\mathrm{P}$ & ALTA PELIGROSIDAD & $K .2 .9$ \\
\hline $\bar{R}$ & RESIDENCIAL & K.2.10 \\
\hline R-1 & Unifamiliar y bifamiliar & \\
\hline$\overline{\mathrm{R}-2}$ & Multifamiliar & \\
\hline R-3 & Hoteles & \\
\hline$T$ & TEMPORAL & K.2.11 \\
\hline
\end{tabular}

Figura. 3 Grupos y Subgrupos de Ocupación. (NSR, 2010)

\begin{tabular}{|l|}
\hline Universidades \\
\hline Colegios \\
\hline Escuelas \\
\hline Centros de Educación \\
\hline Academias \\
\hline Jardines infantiles \\
\hline Otras instituciones docentes \\
\hline
\end{tabular}

Figura. 4 Subgrupos de Ocupación Institucional de Educación. (NSR, 2010)
Con las Empresas Públicas de Armenia se inspeccionaron las tomas fijas para bomberos (siamesas) e hidrantes, revisando entre otros aspectos las presiones y el estado de estos elementos. En la inspección se determinó lo siguiente: 1) La presión de los hidrantes de Plantas Piloto, Facultad de Educación y de la Facultad de Ingeniería se determinó en 32.36 m.c.a. 2) El hidrante ubicado en la Biblioteca Infantil en el momento del ensayo no poseía ningún caudal; por lo tanto se infiere que éste no está conectado a la red hidráulica principal que abastece la institución; por consiguiente se sugiere una revisión a la red. y 3) Los juegos de siamesas ubicados en la Facultad de Educación, Biblioteca Euclides Jaramillo Arango y Facultad de Ciencias de la Salud no se ensayaron ya que no se tenía disponible el equipo especializado; pero según la revisión visual, están completas y en buen estado.

Con el fin de establecer la percepción de la comunidad universitaria sobre las rutas de evacuación en caso de incendio y el grado de conocimiento sobre la existencia de la brigada de emergencia de la institución, se aplicó una encuesta en asesoría de la Oficina de Estadística de la Universidad del Quindío, aplicada a 256 personas de una población de 7904, para un erro de muestreo del 6\% y una confianza del $95 \%$.

Para la pregunta ¿Conoce las rutas de evacuación en caso de incendio en su bloque?, se encontró (Figura. 7) que el $73 \%$ de las personas encuestadas no saben hacia donde evacuar en caso de inicio o totalidad de incendio, mientras que un solo $27 \%$ tiene conocimiento de las rutas de evacuación de sus respectivos bloques.

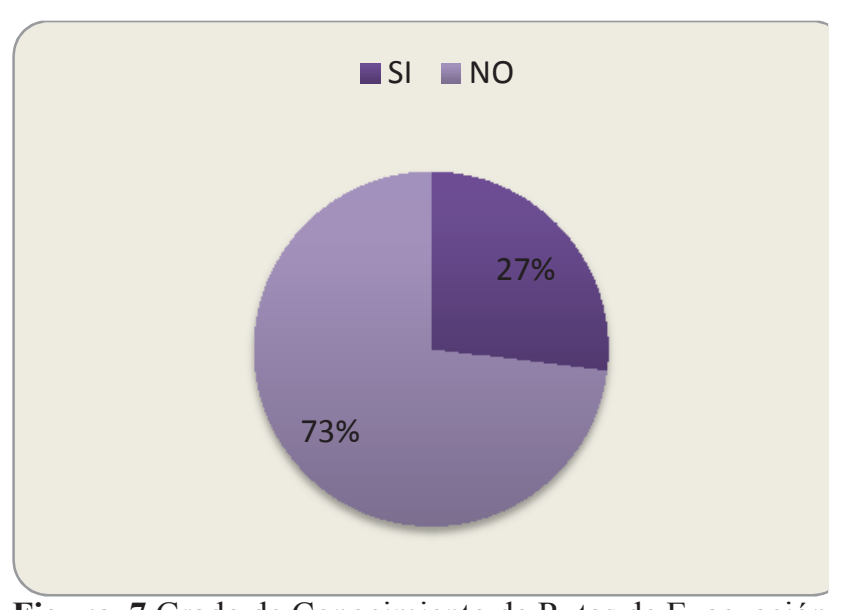

Figura. 7 Grado de Conocimiento de Rutas de Evacuación 


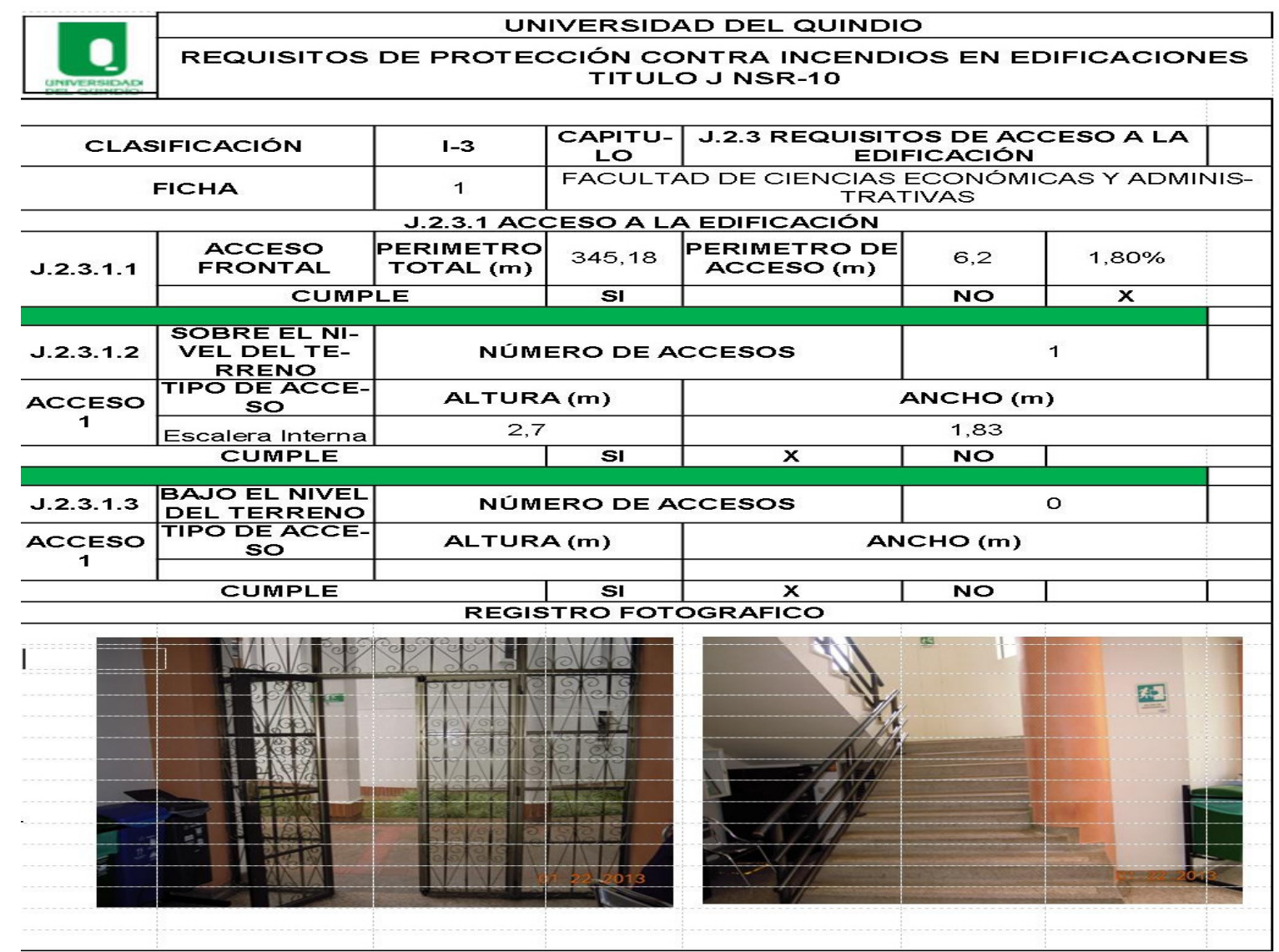

Figura. 5 Evaluación de Accesos.

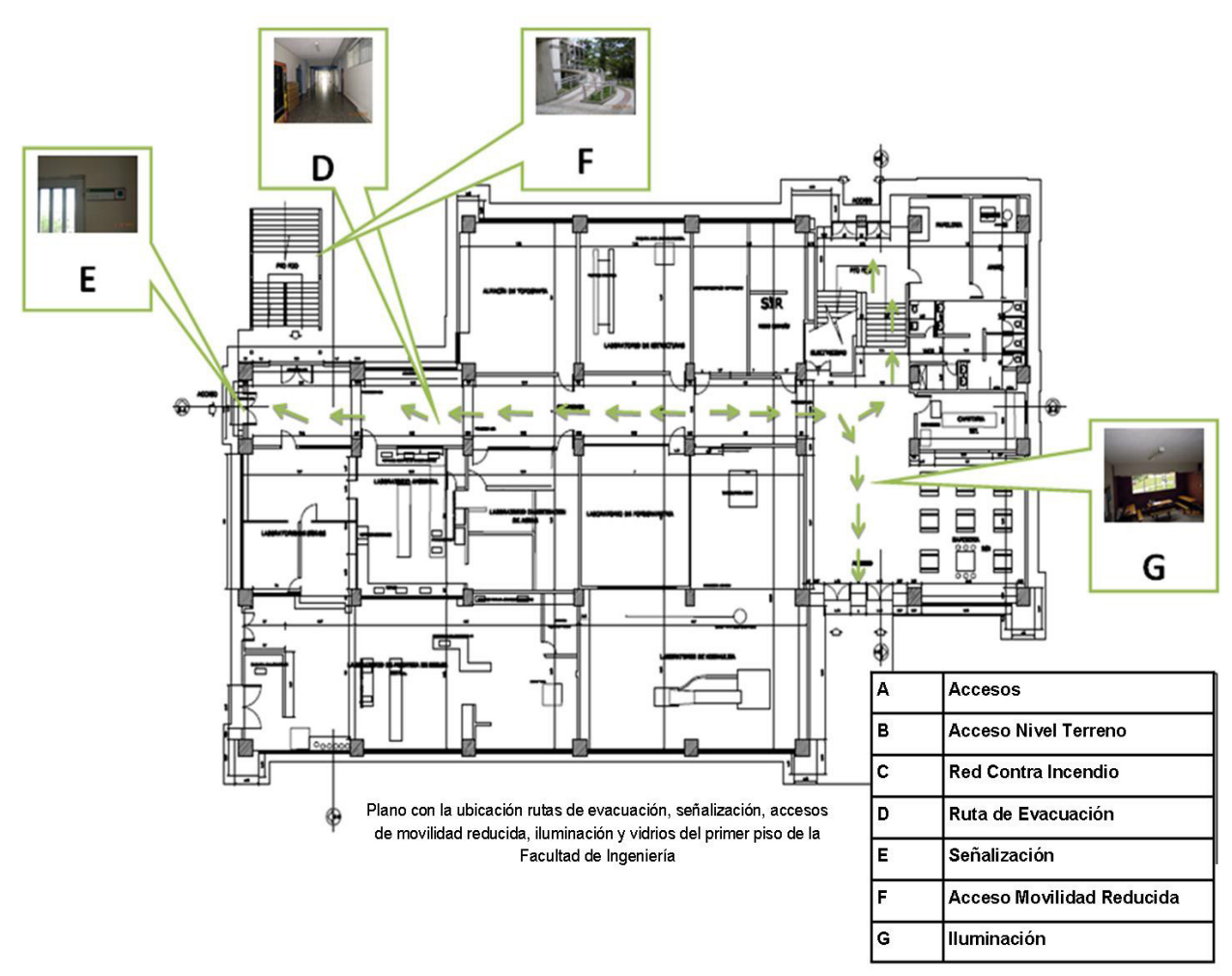

Figura. 6 Rutas de Evacuación 
Para la pregunta ¿Conoce de la existencia de la Brigada de Emergencia de la Universidad? se encontró (Figura. 8) que el $87 \%$ de las personas encuestadas tienen desconocimiento de la existencia de la Brigada de Emergencia de la Universidad del Quindío y sólo el $13 \%$ saben de ella.

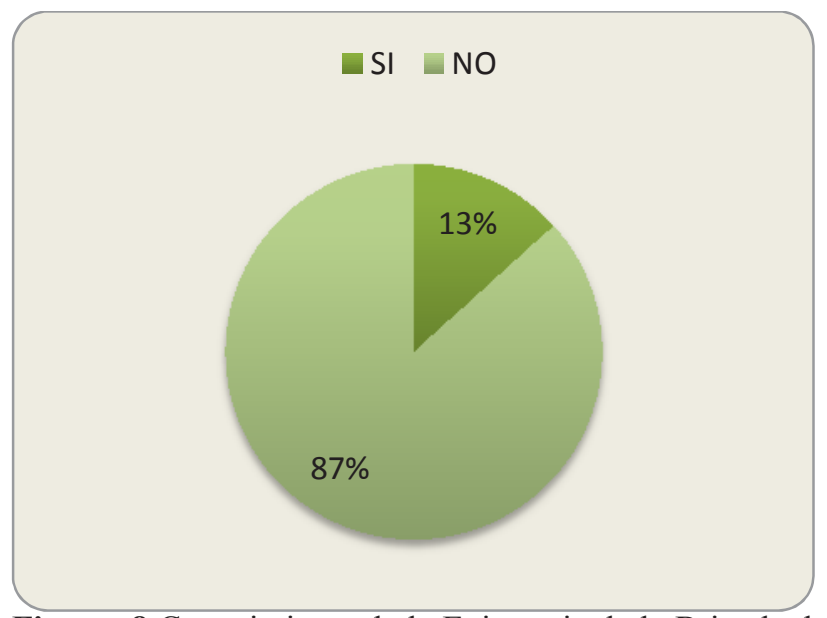

Figura. 8 Conocimiento de la Existencia de la Brigada de Emergencia

Del análisis de resultados se establecieron una serie de recomendaciones, entre las cuales se tienen las siguientes:

Las tomas fijas de agua para bomberos, son insuficientes para cubrir el área en el caso de un incendio. Hay edificaciones que cumplen con el requisito de evacuación, pero se deben mejorar ciertos elementos de la red contra incendio (completar elementos faltantes y la seguridad en algunos gabinetes). Debido a que el funcionamiento de los gabinetes contra incendios no hace parte del desarrollo del proyecto, se recomienda revisar la eficiencia de éstos.

La mayoría de materiales utilizados en acabados interiores son de clase 1 y clase 2 lo que indica muy poco índice de propagación de la llama en caso de un incendio; excepto las divisiones internas en paneles mixtos a media altura, las cuales pueden provocar una propagación muy rápida debido a su alto índice de propagación de la llama (con un índice de más de 225). Se recomienda cambiar los paneles mixtos a media altura por divisiones en material liviano ya que el índice de propagación a la llama de este material es más bajo que el utilizado actualmente en algunas áreas.

Se recomienda en las edificaciones, mantener los ac- cesos sobre el nivel del terreno libre de obstrucciones para facilitar la evacuación en caso de una emergencia.

La mayoría de las escaleras cumplen con las especificaciones requeridas.

Los accesos deben tener su respectiva señalización para mejorar la evacuación. La mayoría de los Bloques de Campus no ilustran las rutas de evacuación, por lo tanto los estudiantes no identifican la ruta adecuada en caso de un evento de incendio.

Algunos Bloques del Campus no tienen señalización en sus entradas, las cuales pueden ser confundidas con un acceso de salida. Se debe colocar señalización en todas las entradas, accesos, corredores y rutas de evacuación en las edificaciones del Campus cumpliendo con los requisitos del título K de la NSR 10.

Se requiere dar a conocer el sistema de evacuación que debe manejar la comunidad universitaria en caso de incendio; esto se logra con una mayor difusión de la Brigada de Emergencia de la Universidad el Quindío, y la aplicación de simulacros y campañas de atención de desastres.

En la visita realizada por el cuerpo oficial de bomberos de la ciudad de Armenia se determinó que es necesaria una inspección a la red hidráulica de la institución, ya que las presiones registradas por el personal de Empresas Públicas de Armenia (EPA) arrojaron valores muy bajos, no adecuados para una edificación de uso educativo.

Por último se recomiendan revisiones con más frecuencia al sistema de la red contra incendio de la universidad; como capacitación al personal de vigilancia sobre el conocimiento de la ubicación exacta de elementos como extintores y gabinetes.

\section{CONCLUSIONES}

La reducción de la vulnerabilidad a un efecto adverso en cualquier edificación, implica niveles de protección diferentes. El nivel más básico lo constituye la protección de vidas humanas, por lo tanto se debe asegurar que los ocupantes de una edificación estén protegidos y puedan evacuar a tiempo.

Según el análisis realizado a las edificaciones que componen la institución educativa, se determinó que 
los bloques más vulnerables son aquellos en donde manejan residuos químicos, papel en exceso y donde permanece la comunidad infantil; estos sitios corresponden a la Facultad de Ciencias Básicas, Biblioteca Euclides Jaramillo Arango y Biblioteca Infantil Alegría de Aprender. Cabe anotar que el resto de las construcciones poseen un alto índice de vulnerabilidad ya que durante el proceso se encontró que la mayoría de los requisitos exigidos por la Norma Sismo Resistente de la NSR 10 en los títulos J y K no cumplen con las especificaciones establecidas en ellos, dada la antigüedad de los edificaciones con relación a la vigencia de la norma.
Se tiene claro que las edificaciones no fueron construidas con el reglamento con el cual se realizó este proyecto, pero hay situaciones que se pueden acomodar a los requisitos exigidos como las señalizaciones, red contra incendios, iluminación, rutas de evacuación y establecer una cultura de prevención y comportamiento adecuado en la comunidad universitaria ante un incendio. De igual manera el apoyo para realizar el presente estudio desde la Oficina de Planeación de la Universidad, y demás dependencias, ilustra el compromiso e importancia que tiene la Gestión de Riesgo de Desastres para la institución.

\section{BIBLIOGRAFIA}

1. Cardona, Omar Darío. (1991), Evaluación de la Amenaza, la Vulnerabilidad y el Riesgo, Taller Regional de Capacitación para la Administración de Desastres ONAD/PNUD/OPS/ UNDRO, Bogotá, 1991.

2. Ministerio del Interior de Colombia. (1998). Decreto 93 de 1998 Por el cual se adopta el Plan Nacional para la Prevención y Atención de Desastres. Bogotá.

3. Narváez, L; Lavell, A y Pérez, G. (2009). La Gestión del Riesgo de Desastres. Un enfoque basado en procesos. 1ed. Lima, Perú: PREDECAN. ISBN: 978-9972-787-88-1.

4. POSITIVA. (2012). Asistencia técnica en el análisis de riesgos y análisis de vulnerabilidad. Edificio de enfermería Universidad Nacional de Colombia. Bogotá.

5. Reglamento colombiano de Construcción Sismo Resistente NRS-10. (2010). Título J "Requisitos de Protección Contra Incendios en Edificaciones" Asociación Colombiana de Ingeniería Sísmica. Bogotá, Colombia.

6. Reglamento colombiano de Construcción Sismo Resistente NRS-10. (2010). Título K "Requisitos Complementarios". Asociación Colombiana de Ingeniería Sísmica. Bogotá, Colombia.

7. República de Colombia (2012). Ley 1523 de 2012 Por la cual se adopta la política nacional de gestión del riesgo de desastres y se establece el Sistema Nacional de Gestión del Riesgo de Desastres y se dictan otras disposiciones. Bogotá

8. UIP y UNISDR. (2010). Reducción del Riesgo de Desastres: Un Instrumento para alcanzar los Objetivos de Desarrollo del Milenio. Ginebra, Suiza, Setiembre de 2010.

9. USAID/OFDA. (2011). Educación y gestión del riesgo: Una experiencia. para compartir. 1 ed. San José, C.R. : 484 p.; 21 X 27 cm.

10. USAID, OFDA, LAC. (2012). Memorias del Evento. Foro Latinoamericano de Reducción del Riesgo de Desastre en la Educación Superior, (pág. 216). Panamá. 